\title{
RATIONAL APPROXIMATIONS TO VALUES OF BELL POLYNOMIALS AT POINTS INVOLVING EULER'S CONSTANT AND ZETA VALUES
}

\section{KH. HESSAMI PILEHROOD and T. HESSAMI PILEHROOD ${ }^{\bowtie}$}

\author{
(Received 19 November 2010; accepted 1 February 2012)
}

Communicated by J. Shallit

Dedicated to the memory of Alfred van der Poorten

\begin{abstract}
In this paper we present new explicit simultaneous rational approximations which converge subexponentially to the values of the Bell polynomials at the points

$$
\left(\gamma, 1 !(2 a+1) \zeta(2), 2 ! \zeta(3), \ldots,(m-1) !\left(a+1+(-1)^{m} a\right) \zeta(m)\right)
$$

where $m=1,2, \ldots, a, a \in \mathbb{N}, \gamma$ is Euler's constant and $\zeta$ is the Riemann zeta function.

2010 Mathematics subject classification: primary 11J13; secondary 33C60, 11B68, 11M35, 33F10.

Keywords and phrases: Euler constant, zeta value, Bell polynomial, Bernoulli polynomial, Meijer $G$ function, rational approximation, saddle point method.
\end{abstract}

\section{Introduction}

In 2007 Aptekarev and his collaborators (see [1, 13]) discovered a sequence of rational approximations $\tilde{p}_{n} / \tilde{q}_{n}$ converging to Euler's constant

$$
\gamma=\lim _{k \rightarrow \infty}\left(1+\frac{1}{2}+\cdots+\frac{1}{k}-\log k\right)
$$

subexponentially. More precisely, the numerators $\tilde{p}_{n}$ and denominators $\tilde{q}_{n}$ of these approximations are positive integers generated by the recurrence relation

$$
\begin{aligned}
(16 n-15) \tilde{q}_{n+1}= & \left(128 n^{3}+40 n^{2}-82 n-45\right) \tilde{q}_{n} \\
& -n^{2}\left(256 n^{3}-240 n^{2}+64 n-7\right) \tilde{q}_{n-1}+n^{2}(n-1)^{2}(16 n+1) \tilde{q}_{n-2}
\end{aligned}
$$

This research was in part supported by grants no. 89110024 (first author) and no. 89110025 (second author) from the School of Mathematics, Institute for Research in Fundamental Sciences (IPM).

(c) 2012 Australian Mathematical Publishing Association Inc. 1446-7887/2012 \$16.00 
with the initial conditions

$$
\begin{array}{rrr}
\tilde{p}_{0}=0, & \tilde{p}_{1}=2, & \tilde{p}_{2}=31, \\
\tilde{q}_{0}=1, & \tilde{q}_{1}=3, & \tilde{q}_{2}=50
\end{array}
$$

and the asymptotics

$$
\begin{gathered}
\tilde{q}_{n}=(2 n) ! \frac{e^{\sqrt{2 n}}}{\sqrt[4]{n}}\left(\frac{1}{\sqrt{\pi}(4 e)^{3 / 8}}+O\left(n^{-1 / 2}\right)\right), \\
\tilde{p}_{n}-\gamma \tilde{q}_{n}=(2 n) ! \frac{e^{-\sqrt{2 n}}}{\sqrt[4]{n}}\left(\frac{2 \sqrt{\pi}}{(4 e)^{3 / 8}}+O\left(n^{-1 / 2}\right)\right) .
\end{gathered}
$$

The present authors (see [7]) found explicit representations for these sequences:

$$
\tilde{q}_{n}=\sum_{k=0}^{n}\left(\begin{array}{l}
n \\
k
\end{array}\right)^{2}(n+k) !, \quad \tilde{p}_{n}=\sum_{k=0}^{n}\left(\begin{array}{l}
n \\
k
\end{array}\right)^{2}(n+k) !\left(H_{n+k}+2 H_{n-k}-2 H_{k}\right)
$$

where $H_{n}=\sum_{k=1}^{n} 1 / k$ is the $n$th harmonic number and $H_{0}:=0$. Formulas (1.2) imply that $\tilde{q}_{n}$ and $\tilde{p}_{n}$ are integers divisible by $n !$ and $n ! / D_{n}$, respectively. Here $D_{n}$ denotes the least common multiple of the numbers $1,2, \ldots, n$. The linear forms $\tilde{p}_{n}-\gamma \tilde{q}_{n}$ do not tend to zero even after cancelation of their coefficients by the large common factor $n ! / D_{n}$. Therefore this construction does not allow one to prove the irrationality of $\gamma$, which is still an open problem. Nevertheless, the construction (1.1) present good rational approximations to Euler's constant as $n \rightarrow \infty$ :

$$
\frac{\tilde{p}_{n}}{\tilde{q}_{n}}-\gamma=2 \pi e^{-2 \sqrt{2 n}}\left(1+O\left(n^{-1 / 2}\right)\right) .
$$

In 2009 Rivoal (see [12]) found another way of rationally approximating the Euler constant $\gamma$, viewed as $-\Gamma^{\prime}(1)$, where $\Gamma$ is the usual gamma function. His construction is based on the following third-order recurrence:

$$
\begin{aligned}
(n+3)^{2}(8 n+11)(8 n+19) y_{n+3}=( & +3)(8 n+11)\left(24 n^{2}+145 n+215\right) y_{n+2} \\
& -(8 n+27)\left(24 n^{3}+105 n^{2}+124 n+25\right) y_{n+1} \\
& +(n+2)^{2}(8 n+19)(8 n+27) y_{n},
\end{aligned}
$$

which provides two sequences of rational numbers $P_{n}$ and $Q_{n}$ for $n \geq 0$ with the initial values

$$
\begin{array}{ccc}
P_{0}=-1, & P_{1}=4, & P_{2}=77 / 4, \\
Q_{0}=1, & Q_{1}=7, & Q_{2}=65 / 2
\end{array}
$$

such that $P_{n} / Q_{n}$ converges to $\gamma$. The sequences $P_{n}$ and $Q_{n}$ satisfy the inclusions (see [8, Corollary 5])

$$
n ! Q_{n}, \quad n ! D_{n} P_{n} \in \mathbb{Z}
$$


and provide better approximations to $\gamma$,

$$
\left|\frac{P_{n}}{Q_{n}}-\gamma\right| \leq c_{0} e^{-9 / 2 n^{2 / 3}+3 / 2 n^{1 / 3}}, \quad\left|Q_{n}\right|=O\left(e^{3 n^{2 / 3}-n^{1 / 3}}\right),
$$

as $n \rightarrow \infty$. Unfortunately this convergence is not fast enough to imply the irrationality of $\gamma$.

In [12] Rivoal also considered a more general construction based on simultaneous Padé approximants to Euler's functions,

$$
\mathcal{E}_{s}(z)=\int_{0}^{\infty} \frac{\log (t)^{s-1} e^{-t}}{z-t} d t, \quad s \in \mathbb{N}, z \in \mathbb{C} \backslash[0,+\infty) .
$$

This approach allows one, in principle, to find rational approximations to some other constants related to higher derivatives of the gamma function $\Gamma^{(n)}(1)$ for $n \geq 2$. Note that $\Gamma^{(n)}(1)$ can be written as (see $[3$, p. 175])

$$
\Gamma^{(n)}(1)=Y_{n}\left(-\gamma, \zeta(2),-2 ! \zeta(3), \ldots,(-1)^{n}(n-1) ! \zeta(n)\right)
$$

where $Y_{n}$ is the Bell polynomial whose definition and basic properties are given below. Unfortunately the details of this construction rapidly become very complicated and Rivoal presented explicit rational approximations for only two numbers $\gamma$ and

$$
\zeta(2)-\gamma^{2}=\Gamma^{\prime \prime}(1)-2 \Gamma^{\prime}(1)^{2} .
$$

More precisely, Rivoal constructed a sixth-order linear recurrence with polynomial coefficients of degree 25 which has three solutions $\left\{a_{1, n}\right\}_{n \geq 0},\left\{a_{2, n}\right\}_{n \geq 0}$, and $\left\{b_{n}\right\}_{n \geq 0}$ such that $a_{1, n}, a_{2, n}, b_{n} \in(1 /(3 n) !(3 n+2) !) \mathbb{Z}$ and

$$
\left|\gamma-\frac{a_{1, n}}{b_{n}}\right| \ll \frac{1}{n^{3 / 8} b_{n}}, \quad\left|\zeta(2)-\gamma^{2}-\frac{a_{2, n}}{b_{n}}\right| \ll \frac{1}{n^{3 / 8} b_{n}}
$$

and

$$
\left|b_{n}\right| \sim \frac{c_{0}}{n^{3 / 8}} \exp \left(4 \sqrt{2} n^{3 / 4}-5 \sqrt{2} / 8 n^{1 / 4}\right)
$$

as $n \rightarrow \infty$, where $c_{0}$ is some positive constant independent of $n$. Notice that the better inclusions, namely, $n !^{2} b_{n}, D_{n} n !^{2} a_{1, n}, D_{n}^{2} n !^{2} a_{2, n} \in \mathbb{Z}$, were proved in [8, Corollary 6].

Recently (see [6]) the authors gave a new interpretation of Aptekarev's approximations to Euler's constant in terms of Meijer $G$-functions and hypergeometric type series. This enabled them to find new rational approximations to $\gamma$ generated by a second-order inhomogeneous linear recurrence with polynomial coefficients. The denominators and numerators of these approximations are given by the formulas

$$
q_{n}=\sum_{k=0}^{n}\left(\begin{array}{l}
n \\
k
\end{array}\right)^{2} k !, \quad p_{n}=\sum_{k=0}^{n}\left(\begin{array}{l}
n \\
k
\end{array}\right)^{2} k !\left(2 H_{n-k}-H_{k}\right) .
$$


The sequence $\left\{q_{n}\right\}_{n \geq 0}$ satisfies the second-order homogeneous linear recurrence

$$
q_{n+2}-2(n+2) q_{n+1}+(n+1)^{2} q_{n}=0
$$

with $q_{0}=1$ and $q_{1}=2$, and the sequence $\left\{p_{n}\right\}_{n \geq 0}$ is a solution of the second-order inhomogeneous linear recurrence

$$
p_{n+2}-2(n+2) p_{n+1}+(n+1)^{2} p_{n}=-\frac{n}{n+2}
$$

with $p_{0}=0$ and $p_{1}=1$. Moreover,

$$
\frac{p_{n}}{q_{n}}-\gamma=e^{-4 \sqrt{n}}\left(2 \pi+O\left(n^{-1 / 2}\right)\right)
$$

as $n \rightarrow \infty$ and

$$
q_{n}=n ! \frac{e^{2 \sqrt{n}}}{n^{1 / 4}}\left(\frac{1}{2 \sqrt{\pi e}}+O\left(n^{-1 / 2}\right)\right)
$$

as $n \rightarrow \infty$.

In this paper we generalize the constructions from our work in [6] and explicitly present new simultaneous rational approximations converging subexponentially to the values of Bell polynomials at the points of the form

$$
\left(\gamma, 1 !(2 a+1) \zeta(2), 2 ! \zeta(3), \ldots,(m-1) !\left(a+1+(-1)^{m} a\right) \zeta(m)\right)
$$

for $m=1,2, \ldots, a$ and $a \in \mathbb{N}$. Note that our approach is different from that of Rivoal and is based upon the application of Meijer $G$-functions and complex integrals.

We begin by recalling several known facts about the Bell polynomials. The exponential (complete) Bell polynomials (first effectively studied by Bell (see [2]) and named in his honor) are the polynomials $Y_{n}\left(x_{1}, \ldots, x_{n}\right)$ in an infinite number of variables $x_{1}, x_{2}, \ldots$ defined by the formal series expansion (see [3, Section 3.3]):

$$
\exp \left(\sum_{m=1}^{\infty} x_{m} \frac{t^{m}}{m !}\right)=\sum_{n=0}^{\infty} Y_{n}\left(x_{1}, x_{2}, \ldots, x_{n}\right) \frac{t^{n}}{n !} .
$$

An explicit representation of $Y_{n}$ is given by (see [2, p. 264])

$$
Y_{n}\left(x_{1}, \ldots, x_{n}\right)=\sum_{\pi(n)} \frac{n !}{k_{1} ! \cdots k_{n} !}\left(\frac{x_{1}}{1 !}\right)^{k_{1}} \cdots\left(\frac{x_{n}}{n !}\right)^{k_{n}}
$$

where the summation is taken over all partitions $\pi(n)$ of $n$, that is, over all $n$-tuples of nonnegative integers $\left(k_{1}, \ldots, k_{n}\right)$ such that

$$
\sum_{j=1}^{n} j k_{j}=n
$$


The Bell polynomials satisfy the following recurrence relation (see [2, p. 263]):

$$
Y_{n+1}\left(x_{1}, \ldots, x_{n+1}\right)=\sum_{k=0}^{n}\left(\begin{array}{l}
n \\
k
\end{array}\right) x_{k+1} Y_{n-k}\left(x_{1}, \ldots, x_{n-k}\right), \quad n \geq 0, Y_{0}=1,
$$

which implies immediately that the complete Bell polynomials have integer coefficients and therefore

$$
\frac{n !}{1 !^{k_{1}} k_{1} ! 2 ! k^{k_{2}} k_{2} ! \cdots n !^{k_{n}} k_{n} !} \in \mathbb{Z}
$$

for $k_{1}, \ldots, k_{n}$ as above. The first six complete Bell polynomials are as follows:

$$
\begin{gathered}
Y_{0}=1, \quad Y_{1}\left(x_{1}\right)=x_{1}, \quad Y_{2}\left(x_{1}, x_{2}\right)=x_{1}^{2}+x_{2}, \quad Y_{3}\left(x_{1}, x_{2}, x_{3}\right)=x_{1}^{3}+3 x_{1} x_{2}+x_{3}, \\
Y_{4}\left(x_{1}, x_{2}, x_{3}, x_{4}\right)=x_{1}^{4}+6 x_{1}^{2} x_{2}+4 x_{1} x_{3}+3 x_{2}^{2}+x_{4}, \\
Y_{5}\left(x_{1}, x_{2}, x_{3}, x_{4}, x_{5}\right)=x_{1}^{5}+10 x_{1}^{3} x_{2}+10 x_{1}^{2} x_{3}+15 x_{1} x_{2}^{2}+5 x_{1} x_{4}+10 x_{2} x_{3}+x_{5} .
\end{gathered}
$$

Let $x_{1}, x_{2}, \ldots, x_{n}, \ldots, y_{1}, y_{2}, \ldots, y_{n}, \ldots$, be two infinite sequences of independent variables. Then we may easily deduce the addition theorem (see [2, p. 265])

$$
Y_{n}\left(x_{1}+y_{1}, \ldots, x_{n}+y_{n}\right)=[Y(x)+Y(y)]^{n}
$$

from (1.3). In ordinary notation this is equivalent to

$$
Y_{n}\left(x_{1}+y_{1}, \ldots, x_{n}+y_{n}\right)=\sum_{k=0}^{n}\left(\begin{array}{l}
n \\
k
\end{array}\right) Y_{k}\left(x_{1}, \ldots, x_{k}\right) Y_{n-k}\left(y_{1}, \ldots, y_{n-k}\right) .
$$

The Bell polynomials play an important role in taking the $n$th derivative of a composite function. That is, the $n$th derivative of the function $e^{f(x)}$ can be expressed in terms of known quantities by

$$
\left(\frac{d}{d x}\right)^{n} e^{f(x)}=e^{f(x)} \cdot Y_{n}\left(f^{\prime}(x), f^{\prime \prime}(x), \ldots, f^{(n)}(x)\right) .
$$

This formula is also known as Faà di Bruno's formula for the $n$th derivative of the composite function.

As usual, let $(\lambda)_{m}$ be the Pochhammer symbol (or the shifted factorial) defined by $(\lambda)_{0}=1$ and

$$
(\lambda)_{m}=\lambda(\lambda+1) \cdots(\lambda+m-1)
$$

for $m \geq 1$ and let $H_{n}^{(m)}$ be a generalized harmonic number given by $H_{n}^{(m)}=\sum_{k=1}^{n} 1 / k^{m}$ and $H_{n}^{(1)}=H_{n}$.

We are now able to formulate our main result.

Theorem 1.1. Let $a \geq 2$ be an integer. For $\mu=1,2, \ldots, a-1$ and any nonnegative integer $n$ define the following sequences of rational numbers:

$$
q_{n}:=\sum_{k=0}^{n}\left(\begin{array}{l}
n \\
k
\end{array}\right)^{a} k ! \in \mathbb{Z}, \quad p_{n, \mu}:=\sum_{k=0}^{n}\left(\begin{array}{l}
n \\
k
\end{array}\right)^{a} k ! Y_{\mu}\left(r_{1}(k), r_{2}(k), \ldots, r_{\mu}(k)\right) \in \mathbb{Q}
$$


where

$$
r_{m}(k):=(m-1) !\left(a H_{n-k}^{(m)}+(-1)^{m}(a-1) H_{k}^{(m)}\right)
$$

for $k=0,1, \ldots, n$. Let

$$
\alpha_{\mu}:=Y_{\mu}\left(\gamma, 1 !(2 a-1) \zeta(2), 2 ! \zeta(3), \ldots,(\mu-1) !\left(a+(-1)^{\mu}(a-1)\right) \zeta(\mu)\right) .
$$

Suppose that the coefficients $b_{m}(a)$ are defined by the expansion

$$
-a \log \left(1+\sum_{m=1}^{a} \frac{\left(2-\frac{m+1}{a}\right)_{m}}{(m+1) !} z^{m}\right)-\sum_{m=1}^{a} \frac{\left(2-\frac{m}{a}\right)_{m-1}}{m !} z^{m}=\sum_{m=1}^{a} b_{m}(a) z^{m}+O\left(z^{a+1}\right)
$$

for $|z|<1$. In particular,

$$
b_{1}(a)=-a, \quad b_{2}(a)=(1-a) / 2, \quad b_{3}(a)=(1-a)(2 a-3) /(6 a) .
$$

Then for every $\mu=1,2, \ldots, a-1$ there exists a positive constant $c_{\mu}=c_{\mu}(a)$ such that for any nonnegative integer $n$,

$$
\left|p_{n, \mu}-q_{n} \alpha_{\mu}\right| \leq \frac{c_{\mu} n !}{n^{a / 2+1 /(2 a)}} \exp \left(\sum_{m=1}^{a-1}(-1)^{m} b_{m}(a) \cos (2 \pi m / a) n^{1-m / a}\right) .
$$

Moreover, $D_{n}^{\mu} \cdot p_{n, \mu} \in \mathbb{Z}$ where $D_{n}$ denotes the least common multiple of the numbers $1,2, \ldots, n$ and the following asymptotic formula holds:

$$
q_{n}=\frac{n !}{\sqrt{a}(2 \pi)^{(a-1) / 2} n^{a / 2+1 /(2 a)}} \exp \left(\sum_{m=1}^{a}(-1)^{m} b_{m}(a) n^{1-m / a}\right)\left(1+O\left(n^{-1 / a}\right)\right)
$$

as $n \rightarrow \infty$.

The sequences $\left\{p_{n, \mu} / q_{n}\right\}_{n \geq 0}$ for $\mu=1,2, \ldots, a-1$ provide good simultaneous rational approximations converging subexponentially to the numbers $\alpha_{\mu}$. Note that

$$
\alpha_{1}=\gamma, \quad \alpha_{2}=\gamma^{2}+(2 a-1) \zeta(2), \quad \alpha_{3}=\gamma^{3}+(6 a-3) \gamma \zeta(2)+2 \zeta(3), \ldots
$$

Corollary 1.2. Let $a \geq 2$ be an integer. Then for $\mu=1,2, \ldots, a-1$,

$$
\begin{aligned}
\left|\alpha_{\mu}-\frac{p_{n, \mu}}{q_{n}}\right| & \leq c_{\mu} \exp \left(\sum_{m=1}^{a-1}(-1)^{m} b_{m}(a)(\cos (2 \pi m / a)-1) n^{1-m / a}\right) \\
& <\exp \left(a(\cos (2 \pi / a)-1) n^{1-1 / a}(1+o(1))\right)
\end{aligned}
$$

where $c_{\mu}=c_{\mu}(a)$ is a positive constant independent of $n$. 
In particular, for Euler's constant we have the following corollary.

Corollary 1.3. Let $a \geq 2$ be an integer. Let the sequence $\left\{q_{n}\right\}_{n \geq 0}$ be defined as in (1.7) and let

$$
p_{n}=\sum_{k=0}^{n}\left(\begin{array}{l}
n \\
k
\end{array}\right)^{a} k !\left(a H_{n-k}-(a-1) H_{k}\right)
$$

for $n \geq 0$. Then

$$
\left|\gamma-\frac{p_{n}}{q_{n}}\right|<e^{a(\cos (2 \pi / a)-1) n^{1-1 / a}(1+o(1))} .
$$

We now consider several examples. For $a=2$ our construction gives the rational approximations to Euler's constant studied in [6].

EXAmple 1.4. For $a=3$ we define three sequences

$$
\begin{aligned}
& q_{n}=\sum_{k=0}^{n}\left(\begin{array}{l}
n \\
k
\end{array}\right)^{3} k !, \quad p_{n, 1}=\sum_{k=0}^{n}\left(\begin{array}{l}
n \\
k
\end{array}\right)^{3} k !\left(3 H_{n-k}-2 H_{k}\right), \\
& p_{n, 2}=\sum_{k=0}^{n}\left(\begin{array}{l}
n \\
k
\end{array}\right)^{3} k !\left(\left(3 H_{n-k}-2 H_{k}\right)^{2}+3 H_{n-k}^{(2)}+2 H_{k}^{(2)}\right) .
\end{aligned}
$$

Then

$$
\left|\gamma-\frac{p_{n, 1}}{q_{n}}\right|<c_{1} e^{-9 / 2 n^{2 / 3}+3 / 2 n^{1 / 3}}, \quad\left|\gamma^{2}+5 \zeta(2)-\frac{p_{n, 2}}{q_{n}}\right|<c_{2} e^{-9 / 2 n^{2 / 3}+3 / 2 n^{1 / 3}},
$$

and

$$
q_{n}=\frac{n !}{n^{5 / 3}} e^{3 n^{2 / 3}-n^{1 / 3}}\left(\frac{e^{1 / 3}}{2 \pi \sqrt{3}}+O\left(n^{-1 / 3}\right)\right)
$$

as $n \rightarrow \infty$. Applying Zeilberger's algorithm of creative telescoping (see [11]), it is possible to show (using the same argument as in [6, Lemma 1]) that the sequences $p_{n, 1}, q_{n}$ are solutions of the third-order homogeneous linear recurrence

$$
\begin{aligned}
(n+1)(8 n-9) f_{n+1}=( & \left.24 n^{3}+13 n^{2}-32 n-18\right) f_{n} \\
& -n\left(24 n^{3}-75 n^{2}+52 n-5\right) f_{n-1}+n(n-1)^{3}(8 n-1) f_{n-2}
\end{aligned}
$$

with initial conditions

$$
\begin{gathered}
p_{0,1}=0, \quad p_{1,1}=1, \quad p_{2,1}=13 / 2, \\
q_{0}=1, \quad q_{1}=2, \quad q_{2}=11,
\end{gathered}
$$

and the sequence $p_{n, 2}$ is a solution of the third-order inhomogeneous linear recurrence

$$
\begin{aligned}
(n+1)(8 n-9) f_{n+1}=( & \left.4 n^{3}+13 n^{2}-32 n-18\right) f_{n}-n\left(24 n^{3}-75 n^{2}+52 n-5\right) f_{n-1} \\
& +n(n-1)^{3}(8 n-1) f_{n-2} \\
+ & 2\left(8 n^{4}-17 n^{3}+74 n^{2}-12 n-9\right) /(n(n+1))
\end{aligned}
$$

with initial values $p_{0,2}=0, p_{1,2}=18, p_{2,2}=95$. 
EXAMPLE 1.5. For $a=4$ put

$$
\begin{gathered}
q_{n}=\sum_{k=0}^{n}\left(\begin{array}{l}
n \\
k
\end{array}\right)^{4} k !, \quad p_{n, 1}=\sum_{k=0}^{n}\left(\begin{array}{l}
n \\
k
\end{array}\right)^{4} k !\left(4 H_{n-k}-3 H_{k}\right), \\
p_{n, 2}=\sum_{k=0}^{n}\left(\begin{array}{l}
n \\
k
\end{array}\right)^{4} k !\left(r_{1}^{2}(k)+r_{2}(k)\right), \quad p_{n, 3}=\sum_{k=0}^{n}\left(\begin{array}{l}
n \\
k
\end{array}\right)^{4} k !\left(r_{1}^{3}(k)+3 r_{1}(k) r_{2}(k)+r_{3}(k)\right),
\end{gathered}
$$

where $r_{m}(k)$ is defined in (1.8). Then

$$
\begin{gathered}
\left|\gamma-\frac{p_{n, 1}}{q_{n}}\right|<c_{1} e^{-4 n^{3 / 4}+3 n^{1 / 2}-5 / 8 n^{1 / 4}}, \quad\left|\gamma^{2}+7 \zeta(2)-\frac{p_{n, 2}}{q_{n}}\right|<c_{2} e^{-4 n^{3 / 4}+3 n^{1 / 2}-5 / 8 n^{1 / 4}}, \\
\left|\gamma^{3}+21 \gamma \zeta(2)+2 \zeta(3)-\frac{p_{n, 3}}{q_{n}}\right|<c_{3} e^{-4 n^{3 / 4}+3 n^{1 / 2}-5 / 8 n^{1 / 4}}
\end{gathered}
$$

and

$$
q_{n}=\frac{c_{0} n !}{n^{17 / 8}} e^{4 n^{3 / 4}-3 / 2 n^{1 / 2}+5 / 8 n^{1 / 4}}\left(1+O\left(n^{-1 / 4}\right)\right)
$$

as $n \rightarrow \infty$. Applying Zeilberger's algorithm of creative telescoping, it is easy to show that the sequences $q_{n}, p_{n, 1}$ and $p_{n, 2}$ satisfy the fourth-order homogeneous linear recurrence

$$
\begin{aligned}
(n+2)^{2} & \left(729 n^{4}-162 n^{3}-171 n^{2}-4 n+6\right) f_{n+2} \\
= & \left(2916 n^{7}+14661 n^{6}+20862 n^{5}+947 n^{4}-13008 n^{3}-2370 n^{2}\right. \\
& +1320 n+312) f_{n+1}-\left(4374 n^{8}-18468 n^{7}-82674 n^{6}-85776 n^{5}\right. \\
& \left.-13062 n^{4}+24204 n^{3}+13528 n^{2}+2680 n+168\right) f_{n} \\
& +n^{2}\left(2916 n^{7}+28512 n^{6}+61848 n^{5}+37667 n^{4}-12898 n^{3}\right. \\
& \left.-17463 n^{2}-2692 n+398\right) f_{n-1}-n^{2}(n-1)^{4} \\
& \times\left(729 n^{4}+2754 n^{3}+3717 n^{2}+2084 n+398\right) f_{n-2}
\end{aligned}
$$

with the initial conditions

$$
\begin{array}{cll}
q_{0}=1, \quad q_{1}=2, & q_{2}=19, & q_{3}=250, \\
p_{0,1}=0, \quad p_{1,1}=1, \quad p_{2,1}=13, & p_{3,1}=409 / 3, \\
p_{0,2}=0, \quad p_{1,2}=32, \quad p_{2,2}=217, & p_{3,2}=26444 / 9,
\end{array}
$$

and the sequence $p_{n, 3}$ satisfies the fourth-order inhomogeneous linear recurrence

$$
\begin{aligned}
(n+2)^{2} & \left(729 n^{4}-162 n^{3}-171 n^{2}-4 n+6\right) f_{n+2} \\
= & \left(2916 n^{7}+14661 n^{6}+20862 n^{5}+947 n^{4}-13008 n^{3}-2370 n^{2}\right. \\
& +1320 n+312) f_{n+1}-\left(4374 n^{8}-18468 n^{7}-82674 n^{6}-85776 n^{5}\right. \\
& \left.-13062 n^{4}+24204 n^{3}+13528 n^{2}+2680 n+168\right) f_{n} \\
& +n^{2}\left(2916 n^{7}+28512 n^{6}+61848 n^{5}+37667 n^{4}-12898 n^{3}\right.
\end{aligned}
$$




$$
\begin{aligned}
& \left.-17463 n^{2}-2692 n+398\right) f_{n-1}-n^{2}(n-1)^{4}\left(729 n^{4}+2754 n^{3}\right. \\
& \left.+3717 n^{2}+2084 n+398\right) f_{n-2}-6\left(729 n^{10}+2754 n^{9}-17424 n^{8}\right. \\
& -179680 n^{7}-490669 n^{6}-549106 n^{5}-194460 n^{4}+100424 n^{3} \\
& \left.+105332 n^{2}+30840 n+3184\right) /\left(n(n+1)^{2}(n+2)\right)
\end{aligned}
$$

with the initial values $p_{0,3}=0, p_{1,3}=60, p_{2,3}=402$ and $p_{3,3}=50761 / 9$.

\section{Analytical construction}

Let $a \geq 2$ be an integer. We consider the function

$$
F(n, t)=\frac{n !^{a}}{\Gamma^{a-1}(t+1) \Gamma^{a}(n-t+1)}
$$

for $n=0,1,2, \ldots$, and, for each integer $\mu$ with $0 \leq \mu \leq a-1$,

$$
F_{n, \mu}:=F_{n, \mu, a}:=\left.\sum_{k=0}^{n}\left(\frac{d}{d t}\right)^{\mu} F(n, t)\right|_{t=k} .
$$

Lemma 2.1. Let $a \geq 2$ be an integer. Then for each $\mu=1,2, \ldots, a-1$ and $v=1, \ldots, \mu$ there exist constants $\lambda_{\mu, v}$ which are independent of $n$ such that for $n=0,1,2, \ldots$,

$$
p_{n, \mu}-q_{n} \alpha_{\mu}=\sum_{\nu=1}^{\mu} \lambda_{\mu, \nu} F_{n, \nu},
$$

$F_{n, 0}=q_{n}$ and $D_{n}^{\mu} \cdot p_{n, \mu} \in \mathbb{Z}$. Here the sequences $q_{n}$ and $p_{n, \mu}$ are defined as in (1.7).

Proof. We begin by defining the function

$$
f(t):=a \log n !-a \log \Gamma(n+1-t)-(a-1) \log \Gamma(t+1)
$$

for $0 \leq t \leq n$. Then we see that

$$
f^{\prime}(t)=a \psi(n+1-t)-(a-1) \psi(t+1)
$$

where $\psi(z)=\Gamma^{\prime}(z) / \Gamma(z)$ is the logarithmic derivative of the gamma function (also known as the digamma function) and, for $m \geq 2$,

$$
f^{(m)}(t)=(-1)^{m-1} a \psi^{(m-1)}(n+1-t)-(a-1) \psi^{(m-1)}(t+1) .
$$

Using the well-known formula for the derivatives of $\psi(t)$,

$$
\psi^{(m)}(t)=(-1)^{m+1} m ! \zeta(m+1, t)
$$

for $m \in \mathbb{N}$, where

$$
\zeta(s, t)=\sum_{j=0}^{\infty} \frac{1}{(j+t)^{s}}
$$

is the Hurwitz zeta function, we may deduce that

$$
f^{(m)}(t)=(-1)^{m}(m-1) !\left((-1)^{m-1} a \zeta(m, n+1-t)-(a-1) \zeta(m, t+1)\right)
$$


for $m \geq 2$. We will be interested in the values of $f^{(m)}(t)$ at the integer points $t=k$ for $0 \leq k \leq n$.

When $m=1$ we may use the well-known properties of the digamma function

$$
\psi(1)=-\gamma, \quad \psi(n+1)=H_{n}-\gamma
$$

for $n \in \mathbb{N}$ and equation (2.1) to deduce that

$$
f^{\prime}(k)=-\gamma+a H_{n-k}-(a-1) H_{k} .
$$

It now follows from equations (2.2) and (2.3) that for any integer $m \geq 2$,

$$
f^{(m)}(k)=(m-1) !\left(\left((-1)^{m-1}(a-1)-a\right) \zeta(m)+a H_{n-k}^{(m)}+(-1)^{m}(a-1) H_{k}^{(m)}\right) .
$$

Now using the definition of $r_{m}(k)$ from formula (1.8), we see that $D_{n}^{m} \cdot r_{m}(k) \in \mathbb{Z}$ and, moreover, from equations (2.4) and (2.5) we may deduce that

$$
r_{m}(k)= \begin{cases}f^{\prime}(k)+\gamma & \text { if } m=1 \\ f^{(m)}(k)+(m-1) !\left(a+(-1)^{m}(a-1)\right) \zeta(m) & \text { if } m \geq 2\end{cases}
$$

Now notice that $F(n, t)=e^{f(t)}$. In order to calculate the $\mu$ th derivative of $F(n, t)$ we can apply the Faà di Bruno formula (1.6) to obtain

$$
\left(\frac{d}{d t}\right)^{\mu} F(n, t)=e^{f(t)} \cdot Y_{\mu}\left(f^{\prime}(t), f^{\prime \prime}(t), \ldots, f^{(\mu)}(t)\right)
$$

for $1 \leq \mu \leq a-1$. Now we apply the addition formula (1.5) to calculate

$$
\begin{aligned}
Y_{\mu}\left(r_{1}(k), r_{2}(k), \ldots, r_{\mu}(k)\right)=\sum_{v=0}^{\mu}\left(\begin{array}{l}
\mu \\
v
\end{array}\right) & Y_{v}\left(f^{\prime}(k), f^{\prime \prime}(k), \ldots, f^{(v)}(k)\right) \\
& \times Y_{\mu-v}(\gamma, 1 !(2 a-1) \zeta(2), 2 ! \zeta(3), \ldots,(\mu-v-1) ! \\
& \left.\times\left(a+(-1)^{\mu-v}(a-1)\right) \zeta(\mu-v)\right) .
\end{aligned}
$$

This implies that

$$
\begin{aligned}
Y_{\mu}\left(r_{1}(k), r_{2}(k), \ldots, r_{\mu}(k)\right)-Y_{\mu}\left(\gamma, 1 !(2 a-1) \zeta(2), \ldots,(\mu-1) !\left(a+(-1)^{\mu}(a-1)\right) \zeta(\mu)\right) \\
=\sum_{v=1}^{\mu}\left(\begin{array}{l}
\mu \\
v
\end{array}\right) Y_{v}\left(f^{\prime}(k), f^{\prime \prime}(k), \ldots, f^{(v)}(k)\right) \\
\quad \times Y_{\mu-v}\left(\gamma, 1 !(2 a-1) \zeta(2), \ldots,(\mu-v-1) !\left(a+(-1)^{\mu-v}(a-1)\right) \zeta(\mu-v)\right)
\end{aligned}
$$

for $0 \leq k \leq n$. Now multiplying both sides of the last equality by $e^{f(k)}=k !\left(\begin{array}{l}n \\ k\end{array}\right)^{a}$ and summing over $k=0,1,2, \ldots, n$,

$$
\begin{aligned}
p_{n, \mu}- & q_{n} \cdot Y_{\mu}\left(\gamma, 1 !(2 a-1) \zeta(2), \ldots,(\mu-1) !\left(a+(-1)^{\mu}(a-1)\right) \zeta(\mu)\right) \\
= & \sum_{v=1}^{\mu}\left(\begin{array}{l}
\mu \\
v
\end{array}\right) Y_{\mu-v}\left(\gamma, 1 !(2 a-1) \zeta(2), \ldots,(\mu-v-1) !\left(a+(-1)^{\mu-v}(a-1)\right) \zeta(\mu-v)\right) \\
& \times\left.\sum_{k=0}^{n}\left(\frac{d}{d t}\right)^{v} F(n, t)\right|_{t=k}
\end{aligned}
$$


or

$$
p_{n, \mu}-q_{n} \alpha_{\mu}=\sum_{v=1}^{\mu} \lambda_{\mu, v} F_{n, v}
$$

where $q_{n}$ and $p_{n, \mu}$ are defined as in (1.7) and the constants $\lambda_{\mu, v}$ given by

$$
\lambda_{\mu, v}=\left(\begin{array}{l}
\mu \\
v
\end{array}\right) \cdot Y_{\mu-v}\left(\gamma, 1 !(2 a-1) \zeta(2), \ldots,(\mu-v-1) !\left(a+(-1)^{\mu-v}(a-1)\right) \zeta(\mu-v)\right)
$$

are independent of $n$.

To prove that $D_{n}^{\mu} \cdot p_{n, \mu} \in \mathbb{Z}$ we consider an arbitrary monomial of the expression $D_{n}^{\mu} \cdot Y_{\mu}\left(r_{1}(k), \ldots, r_{m}(k)\right)$ which has the form

$$
\begin{aligned}
D_{n}^{\mu} & \frac{\mu !}{k_{1} ! \cdots k_{\mu} !}\left(\frac{r_{1}(k)}{1 !}\right)^{k_{1}} \cdots\left(\frac{r_{\mu}(k)}{\mu !}\right)^{k_{\mu}} \\
& =\frac{\mu !}{1 !^{k_{1}} k_{1} ! \cdots \mu !^{k_{\mu}} k_{\mu} !}\left(D_{n} \cdot r_{1}(k)\right)^{k_{1}} \cdot\left(D_{n}^{2} \cdot r_{2}(k)\right)^{k_{2}} \cdots\left(D_{n}^{\mu} \cdot r_{\mu}(k)\right)^{k_{\mu}}
\end{aligned}
$$

where $k_{1}, \ldots, k_{\mu}$ are nonnegative integers such that

$$
1 \cdot k_{1}+2 \cdot k_{2}+3 \cdot k_{3}+\cdots+\mu \cdot k_{\mu}=\mu .
$$

Now by (1.4) and the fact that $D_{n}^{m} \cdot r_{m}(k) \in \mathbb{Z}$ we deduce that $D_{n}^{\mu} \cdot p_{n, \mu} \in \mathbb{Z}$.

Now for each integer $\mu$ with $0 \leq \mu \leq a-1$ and $u \in \mathbb{R}$ we define a complex integral

$$
\begin{aligned}
I_{n, \mu}(u) & :=\frac{1}{2 \pi i} \int_{L} F(n, t)\left(\frac{\pi}{\sin \pi t}\right)^{\mu+1} e^{i \pi t u} d t \\
& =(-1)^{(\mu+1) n} n !^{a} G_{a, a-1}^{0, \mu+1}\left(\begin{array}{c}
n+1, \ldots, n+1 \\
0, \ldots, 0
\end{array} \mid e^{i \pi u}\right)
\end{aligned}
$$

where $L$ is a loop beginning and ending at $-\infty$ and encircling the points $n, n-1$, $n-2, \ldots$ once in the positive direction. Without loss of generality we can assume that $L$ is located symmetrically with respect to the real axis. The integral converges according to the definition of the Meijer $G$-function (see [9, Section 5.2]). Moreover, if $\mu=a-1$ and $|u|<1 / 2$ we can also choose the contour of integration to be a vertical line going from $c-i \infty$ to $c+i \infty$ where $c>n$ is an arbitrary constant.

Let us also define

$$
\widetilde{I}_{n, \mu}(u):=\sum_{k=0}^{n} \operatorname{res}_{t=k}\left(F(n, t)\left(\frac{\pi}{\sin \pi t}\right)^{\mu+1} e^{i \pi t u}\right)
$$

for $0 \leq \mu \leq a-1$.

Lemma 2.2. Let $a \geq 2$ and $0 \leq \mu \leq a-1$ be integers. Then

$$
\widetilde{I}_{n, \mu}(u)= \begin{cases}I_{n, \mu}(u) & \text { if } 0 \leq \mu \leq a-2, \\ I_{n, \mu}(u)+O\left(n^{-a}\right) & \text { if } \mu=a-1,\end{cases}
$$

where the constant in $O$ is absolute. 
Proof. First note that

$$
F(n, t)\left(\frac{\pi}{\sin \pi t}\right)^{\mu+1} e^{i \pi t u}=\frac{(-1)^{n a} n !^{a}}{\pi^{a-\mu-1}} \Gamma(t-n)\left(\frac{\Gamma(t-n)}{\Gamma(t+1)}\right)^{a-1}(\sin \pi t)^{a-\mu-1} e^{i \pi t u} .
$$

Then note that for $t$ lying in the segment $\operatorname{Re} t=-N-1 / 2,|\operatorname{Im} t| \leq y_{0}$ where $N$ is a sufficiently large integer,

$$
\begin{gathered}
|\Gamma(t-n)| \leq|\Gamma(\operatorname{Re}(t-n))|=|\Gamma(-N-1 / 2-n)|=\frac{\pi}{\Gamma(N+1 / 2+n)}=O\left(e^{-N \log N+N}\right), \\
\left|\frac{\Gamma(t-n)}{\Gamma(t+1)}\right|=O\left(\frac{1}{N^{n+1}}\right), \quad\left|(\sin \pi t)^{a-\mu-1} e^{i \pi t u}\right|=O(1) .
\end{gathered}
$$

From this we conclude that the integral $I_{n, \mu}$ can be evaluated as a sum of residues at the singular points lying inside the loop $L$. The integrand in (2.6) has poles of order $\mu+1$ at the points $0,1, \ldots, n$ and, moreover, if $\mu=a-1$, then it has additional simple poles at the negative integers. Therefore

$$
\begin{aligned}
I_{n, a-1}(u) & =\widetilde{I}_{n, a-1}(u)+(-1)^{a n} n !^{a} \sum_{k<0} \operatorname{res}_{t=k}\left(\frac{\Gamma(t-n) e^{i \pi t u}}{(t(t-1) \cdots(t-n))^{a-1}}\right) \\
& =\widetilde{I}_{n, a-1}(u)+(-1)^{a n} n !^{a} \sum_{k<0} \operatorname{res}_{t=k}\left(\frac{e^{i \pi t u}}{\Gamma(n-t+1)(t(t-1) \cdots(t-n))^{a-1}} \frac{\pi}{\sin \pi t}\right) \\
& =\widetilde{I}_{n, a-1}(u)+n !^{a} \sum_{k=1}^{\infty} \frac{(-1)^{(u+1) k+a-1}}{(n+k) !(k(k+1) \cdots(k+n))^{a-1}} \\
& =\widetilde{I}_{n, a-1}(u)+\frac{1}{(n+1)^{a}} \sum_{k=0}^{\infty} \frac{(-1)^{(u+1) k+a-1} k !^{a-1}}{(n+2)_{a}^{k}} .
\end{aligned}
$$

Finally, since

$$
\left|\sum_{k=0}^{\infty} \frac{(-1)^{(u+1) k+a-1} k !^{a-1}}{(n+2)_{a}^{k}}\right| \leq \sum_{k=0}^{\infty} \frac{k !^{a-1}}{(n+2)_{a}^{k}} \leq \sum_{k=0}^{\infty} \frac{1}{k !}=e,
$$

we may deduce our result.

\section{Bernoulli polynomials}

The generalized Bernoulli polynomials $B_{n}^{(m)}(x)$ of order $m$, where $m$ is a positive integer, are defined by the generating formula (see [9, Section 2.8])

$$
\frac{z^{m} e^{x z}}{\left(e^{z}-1\right)^{m}}=\sum_{n=0}^{\infty} B_{n}^{(m)}(x) \frac{z^{n}}{n !}
$$

for $|z|<2 \pi$. Numerous properties of these polynomials can be deduced directly from formula (3.1). We mention only two of them which will be useful in the next section. For a detailed study of Bernoulli polynomials see [10]. 
Comparing powers of $z$ on both sides of the equality

$$
\frac{z^{m} e^{y z}}{\left(e^{z}-1\right)^{m}} \cdot e^{x z}=\frac{z^{m} e^{(y+x) z}}{\left(e^{z}-1\right)^{m}}
$$

leads to the addition formula

$$
B_{n}^{(m)}(x+y)=\sum_{k=0}^{n}\left(\begin{array}{l}
n \\
k
\end{array}\right) B_{k}^{(m)}(y) x^{n-k} .
$$

Differentiating both sides of (3.1) with respect to $z$ and comparing powers of $z$ lets us deduce the recursion formula

$$
m B_{n}^{(m+1)}(x)=(m-n) B_{n}^{(m)}(x)+n(x-m) B_{n-1}^{(m)}(x) .
$$

If we set $n=m$ in (3.3), then we see that $B_{m}^{(m+1)}(x)=(x-m) B_{m-1}^{(m)}(x)$ which implies that

$$
B_{m}^{(m+1)}(x)=(x-1)(x-2) \cdots(x-m) .
$$

LEMMA 3.1.

(1) Let $m$ be a positive integer. Then the following series expansion holds for $|z|<\pi$ :

$$
\frac{z^{m}}{\sin ^{m} z}=\sum_{n=0}^{\infty} \frac{(-1)^{n} 4^{n} B_{2 n}^{(m)}(m / 2)}{(2 n) !} z^{2 n} .
$$

(2) Let $m$ be a positive even integer. Then

$$
\sum_{k=0}^{m}\left(\begin{array}{l}
m \\
k
\end{array}\right) B_{k}^{(m+1)}\left(\frac{m+1}{2}\right) 2^{k}=0 .
$$

Proof. Replacing $z$ by $2 z$ in (3.1) and using the formula $\sinh z=\left(e^{z}-e^{-z}\right) / 2$, we see that, for $|z|<\pi$,

$$
\frac{z^{m} e^{(2 x-m) z}}{\sinh ^{m} z}=\sum_{n=0}^{\infty} B_{n}^{(m)}(x) \frac{2^{n} z^{n}}{n !} .
$$

Setting $x=m / 2$ in the above equality,

$$
\frac{z^{m}}{\sinh ^{m} z}=\sum_{n=0}^{\infty} B_{n}^{(m)}\left(\frac{m}{2}\right) \frac{2^{n} z^{n}}{n !}
$$

for $|z|<\pi$. Since the function $z / \sinh z$ is even, we may deduce that for any positive integer $m$,

$$
B_{2 n+1}^{(m)}\left(\frac{m}{2}\right)=0
$$


for $n=0,1,2, \ldots$ and therefore

$$
\frac{z^{m}}{\sinh ^{m} z}=\sum_{n=0}^{\infty} B_{2 n}^{(m)}\left(\frac{m}{2}\right) \frac{4^{n} z^{2 n}}{(2 n) !}
$$

for $|z|<\pi$. Now replacing $z$ by $i z$ in (3.7) and recalling that $\sin z=-i \sinh (i z)$ gives us the required expansion.

To prove equality (3.5) we consider the addition formula (3.2) with $m$ and $n$ replaced by $m+1$ and $m$, respectively. Setting $x=1 / 2$ and $y=(m+1) / 2$ yields

$$
\sum_{k=0}^{m}\left(\begin{array}{l}
m \\
k
\end{array}\right) B_{k}^{(m+1)}\left(\frac{m+1}{2}\right) 2^{k}=2^{m} B_{m}^{(m+1)}\left(\frac{m+2}{2}\right) .
$$

Now since $m$ is even, applying formula (3.4) lets us deduce that $B_{m}^{(m+1)}(m / 2+1)=0$ and the lemma is proved.

\section{Properties of the integrals $I_{n, \mu}(u)$}

Lemma 4.1. Let $a \geq 2$ and $0 \leq \mu \leq a-1$ be integers. Then

$$
F_{n, \mu}= \begin{cases}\widetilde{I}_{n, 0}(1) & \text { if } \mu=0, \\ \sum_{j=0}^{[\mu / 2]} c_{2 j+1, \mu} \widetilde{I}_{n, 2 j+1}(0) & \text { if } \mu \text { is odd }, \\ \sum_{j=1}^{\mu / 2} c_{2 j, \mu} \operatorname{Re} \widetilde{I}_{n, 2 j}(1) & \text { if } \mu \geq 2 \text { is even }\end{cases}
$$

where $c_{\mu, \mu} \neq 0$ and $c_{j, \mu}$ for $0 \leq j \leq \mu$ are real constants which are independent of $n$.

Proof. We proceed by induction on $\mu$. For $\mu=0$ we easily deduce from (2.7) that

$$
\widetilde{I}_{n, 0}(1)=\sum_{k=0}^{n} \operatorname{res}_{t=k}\left(F(n, t) e^{i \pi t} \cdot \frac{\pi}{\sin \pi t}\right)=\sum_{k=0}^{n} F(n, k)=\sum_{k=0}^{n}\left(\begin{array}{l}
n \\
k
\end{array}\right)^{a} k !=F_{n, 0} .
$$

Similarly, for $\mu=1$,

$$
\widetilde{I}_{n, 1}(0)=\sum_{k=0}^{n} \operatorname{res}\left(F(n, t) \cdot\left(\frac{\pi}{\sin \pi t}\right)^{2}\right)=\left.\sum_{k=0}^{n} \frac{d}{d t} F(n, t)\right|_{t=k}=F_{n, 1} .
$$

For $\mu=2$, applying equation (2.7) allows us to deduce that

$$
\widetilde{I}_{n, 2}(1)=\sum_{k=0}^{n} \operatorname{res}_{t=k}\left(F(n, t) e^{i \pi t} \cdot\left(\frac{\pi}{\sin \pi t}\right)^{3}\right)
$$


Then using the expansions (see Lemma 3.1)

$$
\begin{gathered}
\left(\frac{\pi}{\sin \pi t}\right)^{\mu+1}=(-1)^{(\mu+1) k} \sum_{j=0}^{[\mu / 2]} \frac{(-1)^{j}(2 \pi)^{2 j} B_{2 j}^{(\mu+1)}\left(\frac{\mu+1}{2}\right)}{(2 j) !(t-k)^{\mu+1-2 j}}+O(1), \\
e^{i \pi t}=e^{i \pi k} e^{i \pi(t-k)}=(-1)^{k} \sum_{j=0}^{\mu} \frac{(i \pi)^{j}}{j !}(t-k)^{j}+O\left((t-k)^{\mu+1}\right), \\
F(n, t)=\left.\sum_{j=0}^{\mu} \frac{d^{j}}{d t^{j}} F(n, t)\right|_{t=k} \frac{(t-k)^{j}}{j !}+O\left((t-k)^{\mu+1}\right)
\end{gathered}
$$

in a neighborhood of the integer point $t=k$,

$$
\operatorname{res}_{t=k}\left(F(n, t) e^{i \pi t} \cdot\left(\frac{\pi}{\sin \pi t}\right)^{3}\right)=\left.\frac{1}{2} \frac{d^{2}}{d t^{2}} F(n, t)\right|_{t=k}+\left.i \pi \frac{d}{d t} F(n, t)\right|_{t=k} .
$$

It follows that $F_{n, 2}=2 \operatorname{Re} \widetilde{I}_{n, 2}(1)$.

Now assume that $\mu>2$ and our formula holds for $0,1,2, \ldots, \mu-1$. We prove that our formula holds for $\mu$. If $\mu$ is odd, then

$$
\widetilde{I}_{n, \mu}(0)=\sum_{k=0}^{n} \operatorname{res}_{t=k}\left(F(n, t) \cdot\left(\frac{\pi}{\sin \pi t}\right)^{\mu+1}\right)
$$

and from equations (4.1) and (4.2) we may deduce that

$$
\widetilde{I}_{n, \mu}(0)=\frac{1}{\mu !} F_{n, \mu}+\sum_{j=1}^{[\mu / 2]} d_{2 j, \mu} F_{n, \mu-2 j}
$$

Hence, by our inductive hypothesis, we conclude that

$$
F_{n, \mu}=\mu ! \widetilde{I}_{n, \mu}(0)-\mu ! \sum_{j=1}^{[\mu / 2]} d_{2 j, \mu} F_{n, \mu-2 j}=\mu ! \widetilde{I}_{n, \mu}(0)+\sum_{l=0}^{[\mu / 2]-1} c_{2 l+1, \mu} \widetilde{I}_{n, 2 l+1}(0)
$$

as required.

If $\mu$ is even, then by equation (2.7),

$$
\widetilde{I}_{n, \mu}(1)=\sum_{k=0}^{n} \operatorname{res}_{t=k}\left(F(n, t) e^{i \pi t} \cdot\left(\frac{\pi}{\sin \pi t}\right)^{\mu+1}\right)
$$

and, using the expansions (4.1)-(4.2), we may deduce that

$$
\begin{aligned}
\operatorname{Re} \widetilde{I}_{n, \mu}(1) & =\sum_{j=0}^{\mu / 2} \frac{(-1)^{j}(2 \pi)^{2 j} B_{2 j}^{(\mu+1)}\left(\frac{\mu+1}{2}\right)}{(2 j) !} \sum_{l=0}^{\mu / 2-j} \frac{(-1)^{l} \pi^{2 l}}{(2 l) !(\mu-2 j-2 l) !} F_{n, \mu-2 j-2 l} \\
& =\frac{1}{\mu !} F_{n, \mu}+\sum_{v=1}^{\mu / 2} \widetilde{d}_{2 v, \mu} F_{n, \mu-2 v}
\end{aligned}
$$


where $\widetilde{d}_{2 v, \mu}$ are some real constants independent of $n$ and

$$
\widetilde{d}_{\mu, \mu}=(i \pi)^{\mu} \sum_{j=0}^{\mu / 2} \frac{4^{j} B_{2 j}^{(\mu+1)}\left(\frac{\mu+1}{2}\right)}{(2 j) !(\mu-2 j) !} .
$$

We can apply equation (3.6) to rewrite the last equality as

$$
\widetilde{d}_{\mu, \mu}=(i \pi)^{\mu} \sum_{j=0}^{\mu} \frac{2^{j} B_{j}^{(\mu+1)}\left(\frac{\mu+1}{2}\right)}{j !(\mu-j) !}=\frac{(i \pi)^{\mu}}{\mu !} \sum_{j=0}^{\mu}\left(\begin{array}{c}
\mu \\
j
\end{array}\right) B_{j}^{(\mu+1)}\left(\frac{\mu+1}{2}\right) 2^{j}
$$

which implies that $\widetilde{d}_{\mu, \mu}=0$ by Lemma 3.1 . Now by equation (4.3),

$$
\operatorname{Re} \widetilde{I}_{n, \mu}(1)=\frac{1}{\mu !} F_{n, \mu}+\sum_{v=1}^{\mu / 2-1} \widetilde{d}_{2 v, \mu} F_{n, \mu-2 v}
$$

and the lemma follows by our inductive hypothesis.

Lemma 4.2. Let $a$ and $\mu$ be integers satisfying $a \geq 2,0 \leq \mu \leq a-1$ and $u \in \mathbb{R}$. Then for each $n=0,1,2, \ldots$,

$$
I_{n, \mu}(u)=\frac{1}{(2 \pi i)^{a-\mu-1}} \sum_{k=0}^{a-\mu-1}(-1)^{k}\left(\begin{array}{c}
a-\mu-1 \\
k
\end{array}\right) I_{n, a-1}(a+u-\mu-1-2 k) .
$$

Proof. Equation (2.6) and the reflection formula for the gamma function,

$$
\Gamma(t-n) \Gamma(n-t+1)=\frac{(-1)^{n} \pi}{\sin \pi t},
$$

yield

$$
\begin{aligned}
I_{n, \mu}(u) & =\frac{n !^{a}}{2 \pi i} \int_{L} \frac{e^{i \pi t u}}{\Gamma^{a-1}(t+1) \Gamma^{a}(n-t+1)}\left(\frac{\pi}{\sin \pi t}\right)^{\mu+1} d t \\
& =\frac{(-1)^{a n} n !^{a}}{2 \pi i} \int_{L} \frac{\Gamma^{a}(t-n)}{\Gamma^{a-1}(t+1)}\left(\frac{\sin \pi t}{\pi}\right)^{a-\mu-1} e^{i \pi t u} d t .
\end{aligned}
$$

Now replacing the function $\sin (\pi t)$ by $\left(e^{i \pi t}-e^{-i \pi t}\right) /(2 i)$ and expanding using the binomial theorem, we calculate

$$
I_{n, \mu}(u)=\frac{(-1)^{a n} n !^{a}}{(2 \pi i)^{a-\mu}} \sum_{k=0}^{a-\mu-1}(-1)^{k}\left(\begin{array}{c}
a-\mu-1 \\
k
\end{array}\right) \int_{L} \frac{\Gamma^{a}(t-n)}{\Gamma^{a-1}(t+1)} e^{i \pi t(u+a-\mu-1-2 k)} d t
$$

which completes the proof of the lemma. 
Lemma 4.3. Let $a$ and $\mu$ be integers satisfying $a \geq 2,0 \leq \mu \leq a-1$ and $u \in \mathbb{R}$. Then for each $n=0,1,2, \ldots$,

$$
I_{n, \mu}(-u)=\overline{I_{n, \mu}(u)}
$$

where the bar stands for complex conjugation.

Proof. Making the change of variable $t \mapsto \bar{t}$ in the integral $I_{n, \mu}(u)$ and using the equalities $\Gamma(\bar{z})=\overline{\Gamma(z)}$ and $\sin (\bar{z})=\overline{\sin (z)}$,

$$
\begin{aligned}
& I_{n, \mu}(u)=\frac{1}{2 \pi i} \int_{L} F(n, \bar{t})\left(\frac{\pi}{\sin (\pi \bar{t})}\right)^{\mu+1} e^{i \pi u \bar{t}} d \bar{t}=-\frac{1}{2 \pi i} \int_{L} F(n, \bar{t})\left(\frac{\pi}{\sin (\pi \bar{t})}\right)^{\mu+1} e^{i \pi u \bar{t}} d t \\
& =\overline{\frac{1}{2 \pi i}} \int_{L} \overline{\left(\frac{\pi}{\sin (\pi t)}\right)^{\mu+1}} \overline{F(n, t)} e^{\overline{-i \pi u t}} d t=\overline{\frac{1}{2 \pi i}} \int_{L} \overline{F(n, t)\left(\frac{\pi}{\sin \pi t}\right)^{\mu+1} e^{-i \pi u t}} d t \\
& =\overline{I_{n, \mu}(-u)} \text {, }
\end{aligned}
$$

as required.

\section{Asymptotics of the integral $I_{n, a-1}(u)$}

Lemma 5.1. Let $a, u \in \mathbb{Z}$ satisfy $a \geq 2$ and $|u| \leq a$. Let $n$ be a sufficiently large positive integer. Then all roots of the polynomial

$$
p_{u}(\tau)=e^{i \pi u} n(\tau-1)^{a}-\tau^{a-1}
$$

are given by the following asymptotic expansions for $k=0,1, \ldots, a-1$ :

$$
\tau_{k}(u)=1+\sum_{m=1}^{\infty} \frac{(2-m / a)_{m-1}}{m !} \cdot \frac{e^{i m(2 \pi k-\pi u) / a}}{n^{m / a}} .
$$

Proof. First note that the polynomial $p_{u}(\tau)$ has no real roots on $(-\infty, 0]$. Indeed, if we suppose that $\tau=-x$, where $x \geq 0$, is such a root, then $p_{u}(-x)=0$ and

$$
(-1)^{u} n(x+1)^{a}+x^{a-1}=0 .
$$

On the other hand, the left-hand side of equation (5.1) is positive (negative) if $u$ is even (odd), which is a contradiction.

Therefore it suffices to consider the equation

$$
n(\tau-1)^{a} e^{i \pi u}=\tau^{a-1}
$$

in the complex $\tau$-plane with a cut along the ray $(-\infty, 0]$. It is easy to see that equation (5.2) is equivalent to $a$ relations of the form

$$
n^{1 / a} e^{i(\pi u-2 \pi k) / a}(\tau-1)=\tau^{1-1 / a}
$$

for $k=0,1, \ldots, a-1$ where $\tau^{1-1 / a}=e^{(1-1 / a) \log \tau}$ and we take the principal branch of the logarithm. 
We may immediately deduce from equation (5.3) that all roots of the polynomial $p_{u}(\tau)$ are given by

$$
\tau_{k}(u)=1+\frac{\exp \left(\frac{2 \pi k-\pi u}{a} i\right)}{n^{1 / a}}+\frac{a-1}{a} \cdot \frac{\exp \left(\frac{2(2 \pi k-\pi u)}{a} i\right)}{n^{2 / a}}+O\left(n^{-3 / a}\right)
$$

for $k=0,1, \ldots, a-1$.

It is possible to find a complete asymptotic expansion in (5.4) if we apply the Lagrange inversion formula (see [4, Section 2.2]) to equation (5.3). Indeed, substituting $\tau-1=z$ and rewriting equation (5.3) as

$$
\frac{z}{(z+1)^{1-1 / a}}=n^{-1 / a} \cdot e^{i(2 \pi k-\pi u) / a}
$$

we see that there exist positive numbers $\rho_{1}$ and $\rho_{2}$ such that whenever $n^{-1 / a}<\rho_{1}$ the equation (5.5) has just one solution $z$ in the domain $|z|<\rho_{2}$. That is,

$$
z=\sum_{m=1}^{\infty} \frac{c_{m}}{n^{m / a}} e^{i m(2 \pi k-\pi u) / a}
$$

where the coefficients $c_{m}$ are given by the formula

$$
c_{m}=\left.\frac{1}{m !}\left(\left(\frac{d}{d z}\right)^{m-1}(z+1)^{m(1-1 / a)}\right)\right|_{z=0} .
$$

Suppose that there is another solution of (5.5) with $|z| \geq \rho_{2}$. Then since the function $|z| /(|z|+1)^{1-1 / a}$ increases from 0 to $+\infty$ as $|z|$ increases from 0 to $+\infty$,

$$
\frac{\rho_{2}}{\left(\rho_{2}+1\right)^{1-1 / a}} \leq \frac{|z|}{(|z|+1)^{1-1 / a}} \leq \frac{|z|}{|z+1|^{1-1 / a}}=n^{-1 / a},
$$

which is impossible if $n$ is sufficiently large. Hence formulas (5.6) and (5.7) give the complete asymptotic expansion for $\tau_{k}(u)$. That is,

$$
\tau_{k}(u)=1+\sum_{m=1}^{\infty} \frac{(2-m / a)_{m-1}}{m ! n^{m / a}} e^{i m(2 \pi k-\pi u) / a}
$$

for $k=0,1, \ldots, a-1$ and the lemma is proved.

Lemma 5.2. Let $a, u \in \mathbb{Z}$ satisfy $a \geq 2$ and $|u| \leq a$. Then the following formula holds for the integral $I_{n, a-1}(u)$ as $n \rightarrow \infty$ :

$$
I_{n, a-1}(u)=\frac{(-1)^{u}(2 \pi)^{(a-1) / 2}}{i n^{(a-3) / 2}} \int_{L_{1}} g(\tau) e^{n f(\tau)} d \tau \cdot\left(1+O\left(n^{1 / a-1}\right)\right)
$$

where

$$
\begin{gathered}
f(\tau)=a(\tau-1) \log (\tau-1)-(a-1) \tau \log \tau-\tau+\tau \log n+i \pi \tau u \\
g(\tau)=\frac{(\tau-1)^{a / 2}}{\tau^{3(a-1) / 2}} .
\end{gathered}
$$


If $u \neq 0$, then the contour $L_{1}$ is taken to be a loop beginning and ending at $-\infty$, encircling the points $1,0,-1, \ldots$ once in the positive direction and intersecting the real axis at the point $\tau_{0}:=\tau_{0}(0)$. If $u=0$, then we take $L_{1}$ to be a vertical line going from $\tau_{0}-i \infty$ to $\tau_{0}+i \infty$.

Proof. We choose the branches of the many-valued functions $f(\tau)$ and $g(\tau)$ in the $\tau$ plane with a cut along the ray $(-\infty, 1]$ and fix the branches of the logarithms that take real values on the interval $(1,+\infty)$ of the real axis.

Note that the contour of integration $L$ in the integral $I_{n, a-1}(u)$ defined in equation (2.6) is an arbitrary loop beginning and ending at $-\infty$ and encircling the points $n, n-1, n-2, \ldots$ once in the positive direction or an arbitrary vertical line $\operatorname{Re} \tau=c$ with $c>n$. We now set

$$
\tau_{0}:=\tau_{0}(0)=1+\frac{1}{n^{1 / a}}+O\left(n^{-2 / a}\right)
$$

which is one of the real roots of the polynomial $p_{0}(\tau)$ (see Lemma 5.1) and suppose that the contour $L$ (loop or vertical line) intersects the real axis at the point $n \tau_{0}+1$ and that, for any point $t$ of $L,|t| \geq n \tau_{0}+1$.

Then the asymptotic expansion of the gamma function for large $|z|$ (see [9, Section 2.11]),

$$
\log \Gamma(z)=\left(z-\frac{1}{2}\right) \log z-z+\frac{1}{2} \log (2 \pi)+O\left(|z|^{-1}\right),
$$

where $|\arg z| \leq \pi-\varepsilon, \varepsilon>0$ and the constant in $O$ is independent of $z$, implies that the following formula holds for the integrand of $I_{n, a-1}(u)$ on the contour $L$ :

$$
\begin{aligned}
\frac{\Gamma^{a}(t-n)}{\Gamma^{a-1}(t+1)} e^{i \pi t u}= & \exp \{a \log \Gamma(t-n)-(a-1) \log \Gamma(t+1)+i \pi t u\} \\
= & \exp \left\{a\left(t-n-\frac{1}{2}\right) \log (t-n)-(a-1)\left(t+\frac{1}{2}\right) \log (t+1)+i \pi t u\right. \\
& \left.+a n-t+a-1+\log \sqrt{2 \pi}+O\left(n^{1 / a-1}\right)\right\} .
\end{aligned}
$$

The change of variable $t=n \tau+1$ yields

$$
\begin{aligned}
\frac{\Gamma^{a}(t-n)}{\Gamma^{a-1}(t+1)} e^{i \pi t u} & =(-1)^{u} \sqrt{2 \pi} e^{a n-a n \log n-(a-3 / 2) \log n} \frac{(\tau-1)^{a / 2}}{\tau^{3(a-1) / 2}} e^{n f(\tau)}\left(1+O\left(n^{1 / a-1}\right)\right) \\
& =\frac{(-1)^{u}(2 \pi)^{(a+1) / 2}}{n^{(a-3) / 2} n !^{a}} g(\tau) e^{n f(\tau)}\left(1+O\left(n^{1 / a-1}\right)\right)
\end{aligned}
$$

where the functions $f(\tau)$ and $g(\tau)$ are defined in equations (5.9) and (5.10). This completes the proof of the lemma.

The next lemma is devoted to the calculation of the asymptotics of $I_{n, a-1}(u)$ using the saddle-point method. 
Lemma 5.3. Let $a, u \in \mathbb{Z}$ satisfy $a \geq 2$ and $|u| \leq a$. Then the asymptotic behavior of the integral $I_{n, a-1}(u)$ as $n \rightarrow \infty$ is given by the formula

$$
\begin{aligned}
I_{n, a-1}(u)= & \frac{(-1)^{n u}(2 \pi)^{(a-1) / 2} e^{i \pi u(a-1) /(2 a)}}{\sqrt{a}} \frac{n !}{n^{a / 2+1 /(2 a)}} \\
& \times \exp \left(\sum_{m=1}^{a} b_{m}(a) e^{-i \pi m u / a} n^{1-m / a}\right)\left(1+O\left(n^{-1 / a}\right)\right)
\end{aligned}
$$

where the coefficients $b_{m}(a)$ are rational numbers that can be found explicitly from the expansion (1.9).

Proof. It suffices to prove this lemma only for $u \leq 0$ since we can use Lemma 4.3 to reduce the case when $u>0$ to this case. First we determine the saddle points of the integrand (5.8) which are the zeros of the derivative of the function $f(\tau)$. It is easy to see that the zeros of the derivative

$$
f^{\prime}(\tau)=a \log (\tau-1)-(a-1) \log \tau+\log n+i \pi u
$$

are simultaneously roots of the polynomial $p_{u}(\tau)$ defined in Lemma 4.3.

For the root $\tau_{k}(u)$ where $k=0,1, \ldots, a-1$ we see by Lemma 4.3 that

$$
\tau_{k}(u)-1=\frac{e^{i(2 \pi k-\pi u) / a}}{n^{1 / a}}\left(1+\frac{a-1}{a} \cdot \frac{e^{i(2 \pi k-\pi u) / a}}{n^{1 / a}}+O\left(n^{-2 / a}\right)\right) .
$$

Since

$$
-\frac{\pi u}{a} \leq \frac{2 \pi k-\pi u}{a} \leq-\frac{\pi u}{a}+2 \pi-\frac{2 \pi}{a},
$$

it is easily seen that $\tau_{0}(u)$ is the only zero of the derivative $f^{\prime}(\tau)$ out of all of the roots of equation (5.4). Therefore $\tau_{0}(u)$ is the only saddle point of the function $e^{n f(\tau)}$.

By a similar argument to the one given in the proof of Lemma 4.3 we can find complete asymptotic expansions for the points $\tau$ satisfying the equation $\operatorname{Re} f^{\prime}(\tau)=0$ which is equivalent to

$$
\frac{|z|}{|z+1|^{1-1 / a}}=n^{-1 / a}
$$

where $z=\tau-1$. If we apply the Lagrange inversion formula to the equation

$$
\frac{z}{(z+1)^{1-1 / a}}=w
$$

then we see that there are positive constants $\delta_{1}$ and $\delta_{2}$ such that for $|w|<\delta_{2}$ there is only one solution $z$ to equation (5.12) satisfying $|z|<\delta_{1}$ and this solution is an analytic function of $w$. That is,

$$
z=\sum_{m=1}^{\infty} \frac{(2-m / a)_{m-1}}{m !} w^{m}
$$


Note that by the asymptotic formula (5.11) for the gamma function this series converges if $|w|<a /(a-1)^{1-1 / a}$. So it is clear that if $n>\delta_{2}^{-a}$, then there is one and only one solution of equation (5.12) in the circle $|\tau-1|<\delta_{1}$ given by

$$
\tau=1+\sum_{m=1}^{\infty} \frac{(2-m / a)_{m-1}}{m !} \frac{e^{i m \varphi}}{n^{m / a}}
$$

for $-\pi<\varphi \leq \pi$.

On the other hand, if we suppose that there is another solution with $|z| \geq \delta_{1}$, then the argument used in the proof of Lemma 4.3 gives us a contradiction if $n$ is sufficiently large. So, if $n$ is large enough, the continuously differentiable curve

$$
\operatorname{Re} f^{\prime}(\tau)=\log \frac{|\tau-1|^{a} n}{|\tau|^{a-1}}=0
$$

approximately represents a small circle with center at $\tau=1$ and radius $n^{-1 / a}$. The points of this curve are given by their complete asymptotic expansion (5.13). Moreover, we have $\operatorname{Re} f^{\prime}(\tau)<0$ inside this curve and $\operatorname{Re} f^{\prime}(\tau)>0$ outside it. It is easily seen that $\operatorname{Re} f^{\prime}\left(\tau_{k}(u)\right)=0$ for any integers $u$ and $k$.

To apply the saddle-point method to the evaluation of the integral $I_{n, a-1}(u)$ we need to choose a new contour of integration $L_{2}$ passing through the saddle point $\tau_{0}(u)$. If $u=0$ we use the original contour $\operatorname{Re} \tau=\tau_{0}$. Then for $\tau=\tau_{0}+i y$ with $-\infty<y<+\infty$ the Cauchy-Riemann conditions yield

$$
\frac{d}{d y} \operatorname{Re} f\left(\tau_{0}+i y\right)=-\operatorname{Im} \frac{d}{d \tau} f\left(\tau_{0}+i y\right)=-a \arg (\tau-1)+(a-1) \arg \tau .
$$

Since $\operatorname{Re} \tau_{0}>1$ we see that, for $y<0$,

$$
-\frac{\pi}{2}<\arg (\tau-1)<\arg \tau<0
$$

and therefore

$$
\frac{d}{d y} \operatorname{Re} f\left(\tau_{0}+i y\right)=(a-1)(\arg \tau-\arg (\tau-1))-\arg (\tau-1)>0 .
$$

This implies that $\operatorname{Re} f\left(\tau_{0}+i y\right)$ strictly increases as $y$ increases from $-\infty$ to 0 .

If $y>0$, then

$$
0<\arg \tau<\arg (\tau-1)<\frac{\pi}{2}
$$

and hence

$$
\frac{d}{d y} \operatorname{Re} f\left(\tau_{0}+i y\right)=(a-1)(\arg \tau-\arg (\tau-1))-\arg (\tau-1)<0 .
$$

Therefore the function $\operatorname{Re} f\left(\tau_{0}+i y\right)$ strictly decreases as $y$ increases from 0 to $+\infty$. This proves that $\operatorname{Re} f\left(\tau_{0}+i y\right)$ attains its maximum on $L_{1}$ at the unique point $\tau_{0}$ and we can apply the saddle-point method to calculate the asymptotics of $I_{n, a-1}(0)$ (see (5.21)). 
If $u \neq 0$ we define $L_{2}$ to be a contour consisting of three parts:

(i) the half line $\tau=\tau_{0}+i y$ with $-\infty<y \leq 0$;

(ii) the segment $\tau=\tau_{0}+r e^{i \varphi}$ where $\varphi=\arg \left(\tau_{0}(u)-\tau_{0}\right)$ with $0 \leq r \leq\left|\tau_{0}(u)-\tau_{0}\right|$, connecting the points $\tau_{0}$ and $\tau_{0}(u)$;

(iii) the half line $\tau=-x+i \operatorname{Im} \tau_{0}(u)$ with $-\operatorname{Re} \tau_{0}(u) \leq x<+\infty$.

If $u=-a$, then both parts (ii) and (iii) give a ray going from $\tau_{0}$ to $-\infty$ along the upper bank of the cut $[-\infty, 1]$.

We now show that we can replace the contour $L_{1}$ in the integral $I_{n, a-1}(u)$ by the contour $L_{2}$, that is, we show that

$$
\int_{L_{1}} e^{n f(\tau)} g(\tau) d t=\int_{L_{2}} e^{n f(\tau)} g(\tau) d t .
$$

For this purpose we consider the circle $|\tau|=N$ where $N$ is a sufficiently large integer. Suppose that $L_{1}^{-}, L_{2}^{-}\left(L_{1}^{+}, L_{2}^{+}\right)$are the points of intersection of this circle with the contours $L_{1}$ and $L_{2}$ in the lower half plane (upper half plane), respectively. Then to prove (5.15) it is sufficient to show that

$$
\int_{L_{1}^{-} L_{2}^{-}} e^{n f(\tau)} g(\tau) d t \rightarrow 0, \quad \int_{L_{2}^{+} L_{1}^{+}} e^{n f(\tau)} g(\tau) d t \rightarrow 0
$$

as $N \rightarrow \infty$. Here $L_{1}^{-} L_{2}^{-}$and $L_{2}^{+} L_{1}^{+}$are arcs of the circle of radius $N$ with center at the origin. On the $\operatorname{arcs} L_{1}^{-} L_{2}^{-}$and $L_{2}^{+} L_{1}^{+}$of the circle $\tau=N e^{i \theta}$ for $N$ sufficiently large the inequalities $-\pi<\theta<-\pi / 4$ and $3 \pi / 4<\theta \leq \pi$ respectively hold. The value $\theta=\pi$ corresponds to the upper bank of the cut $(-\infty, 1]$. By Taylor's formula,

$$
\log (\tau-1)=\log \left(N e^{i \theta}-1\right)=\log N+i \theta-\frac{e^{-i \theta}}{N}+O\left(N^{-2}\right)
$$

where the constant in $O\left(N^{-2}\right)$ is absolute. Substituting this expansion in equation (5.9), we see that

$$
\begin{aligned}
\operatorname{Re} f\left(N e^{i \theta}\right)= & N \log N \cos (\theta)+N \log \frac{n}{e} \cos (\theta) \\
& -N(\theta+\pi u) \sin (\theta)-a \log N-a+O\left(N^{-1}\right) .
\end{aligned}
$$

Note that on the $\operatorname{arc} L_{2}^{-} L_{1}^{-}$,

$$
-1 \leq \cos (\theta) \leq \tau_{0} / \sqrt{N^{2}+\tau_{0}^{2}}
$$

$\sin (\theta) \leq 0$ and $\theta+\pi u<0$. This yields

$\operatorname{Re} f(\tau)<\tau_{0} \log N+\tau_{0} \log \frac{n}{e}-a \log N-a+O\left(N^{-1}\right)=\left(\tau_{0}-a\right) \log N+O(1)$.

Similarly, on the $\operatorname{arc} L_{1}^{+} L_{2}^{+}$,

$$
-1 \leq \cos (\theta) \leq-\sqrt{2} / 2
$$


$\sin (\theta) \geq 0$ and, therefore,

$$
\operatorname{Re} f(\tau)<-\frac{\sqrt{2}}{2} N \log N+O(N)
$$

For the function $g(\tau)$ on the $\operatorname{arcs} L_{1}^{-} L_{2}^{-}$and $L_{1}^{+} L_{2}^{+}$we have the trivial estimate

$$
|g(\tau)|=O\left(N^{3 / 2-a}\right)
$$

as $N \rightarrow \infty$.

Since the lengths of each of the arcs $L_{1}^{-} L_{2}^{-}$and $L_{1}^{+} L_{2}^{+}$do not exceed $\pi N$, estimates (5.17)-(5.19) imply that the integrals in (5.16) are of orders $O\left(N^{\tau_{0}+1 / 2-2 a}\right)$ and $O\left(N^{1 / 2-a-\sqrt{2} / 2 N}\right)$, respectively. Hence, the limiting relations in (5.16) hold and we can replace the contour of integration $L_{1}$ in the integral $I_{n, a-1}(u)$ by the new contour $L_{2}$.

We now show that $\tau_{0}(u)$ is the unique maximum point of $\operatorname{Re} f(\tau)$ on the contour $L_{2}$. Since $u<0$, it follows from equation (5.14) that $(d / d y) \operatorname{Re} f\left(\tau_{0}+i y\right)$ is positive when $y<0$, and therefore $\operatorname{Re} f(\tau)$ monotonically increases on the half line $\tau=\tau_{0}+i y$ with $-\infty<y \leq 0$. Similarly, on the half line $\tau=-x+i \operatorname{Im} \tau_{0}(u)$,

$$
\frac{d}{d x} \operatorname{Re} f\left(-x+i \operatorname{Im} \tau_{0}(u)\right)=-\operatorname{Re} \frac{d}{d \tau} f\left(-x+i \operatorname{Im} \tau_{0}(u)\right)<0 .
$$

This shows that the function $\operatorname{Re} f(\tau)$ monotonically decreases on the half line going from $\tau_{0}(u)$ to $-\infty+i \operatorname{Im} \tau_{0}(u)$.

Now consider the segment $\tau=\tau_{0}+r e^{i \varphi}$ defined in (ii). The derivative of $\operatorname{Re} f(\tau)$ on this part of $L_{2}$ is given by the formula

$$
\frac{d}{d r} \operatorname{Re} f(\tau)=\operatorname{Re} \frac{d f(\tau)}{d r}=\operatorname{Re}\left(\frac{d f(\tau)}{d \tau} \cdot \frac{d \tau}{d r}\right)=\operatorname{Re} f^{\prime}(\tau) \cos \varphi-\operatorname{Im} f^{\prime}(\tau) \sin \varphi
$$

Note that since $\operatorname{Re} \tau_{0}(u)<\operatorname{Re} \tau_{0}$, it follows that $\pi / 2<\varphi \leq \pi$ and therefore the product $\operatorname{Re} f^{\prime}(\tau) \cdot \cos \varphi$ is positive on the segment.

Let us investigate the behavior of $\operatorname{Im} f^{\prime}(\tau)$ on this part of the contour. Note that $\operatorname{Im} f^{\prime}\left(\tau_{0}\right)=\pi u<0$ and $\operatorname{Im} f^{\prime}\left(\tau_{0}(u)\right)=0$. We show that $\operatorname{Im} f^{\prime}(\tau)$ monotonically increases on our segment from $\pi u$ to 0 . To see this we consider the derivative of Im $f^{\prime}(\tau)$. By the Cauchy-Riemann conditions, for $r>0$,

$$
\frac{d}{d r} \operatorname{Im} f^{\prime}(\tau)=\operatorname{Im}\left(f^{\prime \prime}(\tau) \cdot e^{i \varphi}\right)=\frac{1}{r} \operatorname{Im}\left(f^{\prime \prime}(\tau) \cdot\left(\tau-\tau_{0}\right)\right) .
$$

Since

$$
f^{\prime \prime}(\tau)=\frac{a}{\tau-1}-\frac{a-1}{\tau}
$$

we obtain

$$
\frac{d}{d r} \operatorname{Im} f^{\prime}(\tau)=\frac{1}{r} \operatorname{Im}\left(\frac{a\left(\tau-\tau_{0}\right)}{\tau-1}-\frac{(a-1)\left(\tau-\tau_{0}\right)}{\tau}\right)=\frac{\operatorname{Im} \tau}{r}\left(\frac{a\left(\tau_{0}-1\right)}{|\tau-1|^{2}}-\frac{(a-1) \tau_{0}}{|\tau|^{2}}\right) .
$$


Since $\tau$ lies in the upper half plane, to show that $(d / d r) \operatorname{Im} f^{\prime}(\tau)$ is positive on our segment, it is sufficient to show that the quantity in parentheses, which we denote by $B$, is positive.

For $\tau=\tau_{0}+r e^{i \varphi}$,

$$
B:=\frac{a\left(\tau_{0}-1\right)}{|\tau-1|^{2}}-\frac{(a-1) \tau_{0}}{|\tau|^{2}}=\frac{h(r)}{|\tau-1|^{2}|\tau|^{2}}
$$

where

$$
h(r)=\left(\tau_{0}-a\right) r^{2}+2 r \tau_{0}\left(\tau_{0}-1\right) \cos \varphi+\tau_{0}\left(\tau_{0}-1\right)\left(a+\tau_{0}-1\right) .
$$

Since $\tau_{0}<a$, it follows easily that the quadratic polynomial $h(r)$ has two real roots $r_{1}$ and $r_{2}$ such that $r_{1}<0<r_{2}$ and $h(r)$ is positive on $\left(r_{1}, r_{2}\right)$ and negative on $\left(-\infty, r_{1}\right)$, $\left(r_{2},+\infty\right)$.

We now show that the point $r(u):=\left|\tau_{0}(u)-\tau_{0}\right|$ belongs to the interval $\left(0, r_{2}\right)$. Indeed, since

$$
\left|\tau_{0}-1\right|=\frac{1}{n^{1 / a}}+O\left(n^{-2 / a}\right) \quad \text { and } \quad\left|\tau_{0}(u)-1\right|=\frac{1}{n^{1 / a}}+O\left(n^{-2 / a}\right)
$$

for $n$ sufficiently large, we have $\left|\tau_{0}(u)-1\right| \leq 2\left|\tau_{0}-1\right|$ and $\tau_{0}<9 / 8$. Therefore

$$
r(u)=\left|\tau_{0}(u)-\tau_{0}\right| \leq\left|\tau_{0}(u)-1\right|+\left|\tau_{0}-1\right| \leq 3\left(\tau_{0}-1\right) .
$$

This implies that

$$
\begin{aligned}
h(r(u)) & \geq 9\left(\tau_{0}-a\right)\left(\tau_{0}-1\right)^{2}-6 \tau_{0}\left(\tau_{0}-1\right)^{2}+\tau_{0}\left(\tau_{0}-1\right)\left(a+\tau_{0}-1\right) \\
& =\left(\tau_{0}-1\right)\left(a\left(9-8 \tau_{0}\right)+4 \tau_{0}\left(\tau_{0}-1\right)\right)>0
\end{aligned}
$$

and hence $r(u) \in\left(0, r_{2}\right)$. This proves that $(d / d r) \operatorname{Im} f^{\prime}(\tau)$ is positive on the segment $[0, r(u)]$ and therefore that $\operatorname{Im} f^{\prime}(\tau)$ increases on this segment from $\pi u$ to 0 , that is, $\operatorname{Im} f^{\prime}(\tau)<0$ on $[0, r(u))$.

Now by (5.20) we see that $(d / d r) \operatorname{Re} f(\tau)>0$ on the segment connecting $\tau_{0}$ and $\tau_{0}(u)$, and therefore $\operatorname{Re} f(\tau)$ is monotonically increasing on this segment. Hence we have shown that $\tau_{0}(u)$ is the unique maximum point on the whole contour of integration $L_{2}$ and we can apply the saddle-point method (see [5, Ch. 6, Theorem 3.1]) to estimate the integral $I_{n, a-1}(u)$.

Recall that when $u=-a$ the contour $L_{2}$ consists of the two parts: the vertical half line $\tau=\tau_{0}+i y,-\infty<y \leq 0$, and the horizontal half line $\tau=-x+i 0$ where $x \geq \operatorname{Re} \tau_{0}(a)$, going along the upper bank of the cut $(-\infty, 1]$. Notice that in this case the function $g(\tau)$ has a singularity at the point $\tau=0$ of the contour $L_{2}$. So, in order to apply the saddle-point method in this case, we need to change the contour $L_{2}$ slightly to avoid this singularity.

It is easy to see that, instead of the horizontal line $\tau=-x+i 0$ with $x \geq-1 / 2$, we can take a contour consisting of the semicircle $\tau=1 / 2 e^{i \varphi}$ for $0 \leq \varphi \leq \pi$ and the ray $\tau=-x+i 0$ with $x \geq 1 / 2$. Then

$$
\int_{L_{2}} e^{n f(\tau)} g(\tau) d t=\int_{\tau_{0}-i \infty}^{\tau_{0}+i 0} e^{n f(\tau)} g(\tau) d t+\int_{\tau_{0}+i 0}^{1 / 2+i 0} e^{n f(\tau)} g(\tau) d t+O\left(C^{n} n^{1 / 2}\right)
$$


where $C=C(a)$ is some positive constant independent of $n$. So, in this case, we can apply the saddle-point method to the contour

$$
L_{2, a}:=\left\{\tau_{0}+i y \mid-\infty<y \leq 0\right\} \cup\left\{x+i 0 \mid 1 / 2 \leq x \leq \tau_{0}\right\}
$$

and, as we can see from equation (5.22) below, the quantity $O\left(C^{n} n^{1 / 2}\right)$ has no influence on the contribution of the saddle point. Finally, applying the saddle-point method, we calculate

$$
\begin{aligned}
I_{n, a-1}(u)=\frac{(-1)^{u}(2 \pi)^{(a / 2)}}{i n^{(a-2) / 2}} e^{(\pi i / 2)-(i / 2) \arg f^{\prime \prime}\left(\tau_{0}(u)\right)}\left|f^{\prime \prime}\left(\tau_{0}(u)\right)\right|^{-1 / 2} \\
\quad \times g\left(\tau_{0}(u)\right) e^{n f\left(\tau_{0}(u)\right)}\left(1+O\left(n^{-1 / a}\right)\right) .
\end{aligned}
$$

In order to find the contribution of the saddle point, we evaluate

$$
\begin{aligned}
f\left(\tau_{0}(u)\right)=- & a \log \left(\tau_{0}(u)-1\right)-\tau_{0}(u)=\pi u i+\log n-1-\sum_{m=1}^{a} \frac{\left(2-\frac{m}{a}\right)_{m-1}}{m !} \frac{e^{-i \pi m и / a}}{n^{m / a}} \\
& -a \log \left(1+\sum_{m=1}^{a} \frac{\left(2-\frac{m+1}{a}\right)_{m}}{(m+1) !} \frac{e^{-i \pi m u / a}}{n^{m / a}}\right)+O\left(n^{-1-1 / a}\right) .
\end{aligned}
$$

Now expanding the logarithm in powers of $e^{-i \pi u / a} n^{-1 / a}$, we see that

$$
\begin{gathered}
-a \log \left(1+\sum_{m=1}^{a} \frac{\left(2-\frac{m+1}{a}\right)_{m}}{(m+1) !} \frac{e^{-i \pi т и / a}}{n^{m / a}}\right)-\sum_{m=1}^{a} \frac{\left(2-\frac{m}{a}\right)_{m-1}}{m !} \frac{e^{-i \pi т и / a}}{n^{m / a}} \\
=\sum_{m=1}^{a} b_{m}(a) \frac{e^{-i \pi m и / a}}{n^{m / a}}+O\left(n^{-1-1 / a}\right)
\end{gathered}
$$

where the $b_{m}(a)$ are rational coefficients depending only on $a$. In particular, $b_{1}(a)=-a$, $b_{2}(a)=(1-a) / 2$ and $b_{3}(a)=(1-a)(2 a-3) /(6 a)$. It follows from equation $(5.22)$ that

$$
e^{n f\left(\tau_{0}(u)\right)}=\frac{(-1)^{u n} n !}{\sqrt{2 \pi n}} \exp \left(\sum_{m=1}^{a} b_{m}(a) e^{-i \pi m u / a} n^{1-m / a}\right)\left(1+O\left(n^{-1 / a}\right)\right) .
$$

Since

$$
g\left(\tau_{0}(u)\right)=e^{-i \pi u / 2} n^{-1 / 2}\left(1+O\left(n^{-1 / a}\right)\right), \quad f^{\prime \prime}\left(\tau_{0}(u)\right)=a e^{i \pi u / a} n^{1 / a}\left(1+O\left(n^{-1 / a}\right)\right),
$$

we obtain the asymptotic behavior of $I_{n, a-1}(u)$ :

$$
\begin{aligned}
I_{n, a-1}(u)= & \frac{(-1)^{n u}(2 \pi)^{(a-1) / 2} e^{i \pi u(a-1) / 2 a}}{\sqrt{a}} \frac{n !}{n^{(a / 2)+(1 / 2 a)}} \\
& \times \exp \left(\sum_{m=1}^{a} b_{m}(a) e^{-i \pi m u / a} n^{1-m / a}\right)\left(1+O\left(n^{-1 / a}\right)\right),
\end{aligned}
$$

and the lemma is proved. 


\section{Proof of Theorem 1.1}

LemMa 6.1. Let $a$ and $\mu$ be positive integers satisfying $a \geq 2$ and $0 \leq \mu \leq a-1$. Then there exist positive constants $\lambda_{0}=\lambda_{0}(a)$ and $\lambda_{1}=\lambda_{1}(a)$ such that for every positive integer $n$,

$$
\left|I_{n, \mu}(0)\right| \leq \frac{\lambda_{0} n !}{n^{a / 2+1 /(2 a)}} \exp \left(\sum_{m=1}^{a-1} b_{m}(a) \cos \left(\frac{\pi m(a-\mu-1)}{a}\right) n^{1-m / a}\right),
$$

and for infinitely many positive integers $n$ a similar lower bound holds. That is,

$$
\left|I_{n, \mu}(0)\right| \geq \frac{\lambda_{1} n !}{n^{a / 2+1 /(2 a)}} \exp \left(\sum_{m=1}^{a-1} b_{m}(a) \cos \left(\frac{\pi m(a-\mu-1)}{a}\right) n^{1-m / a}\right) .
$$

Moreover, the asymptotic formula

$$
\begin{aligned}
\left|I_{n, \mu}(1)\right|= & \frac{n !}{\sqrt{a}(2 \pi)^{((a-1) / 2)-\mu} n^{a / 2+1 /(2 a)}} \\
& \quad \times \exp \left(\sum_{m=1}^{a-1} b_{m}(a) \cos \left(\frac{\pi m(a-\mu)}{a}\right) n^{1-m / a}\right)\left(1+O\left(n^{-1 / a}\right)\right)
\end{aligned}
$$

holds as $n \rightarrow \infty$.

Proof. By Lemma 4.2,

$$
I_{n, \mu}(1)=\frac{1}{(2 \pi i)^{a-\mu-1}} \sum_{k=0}^{a-\mu-1}(-1)^{k}\left(\begin{array}{c}
a-\mu-1 \\
k
\end{array}\right) I_{n, a-1}(a-\mu-2 k) .
$$

It now follows by Lemmas 5.3 and 4.3 that the sum on the right-hand side of equation (6.3) contains exactly one term with dominant asymptotics, that is, $I_{n, a-1}(a-\mu)$. Therefore

$$
\begin{aligned}
\left|I_{n, \mu}(1)\right| \sim & \frac{1}{(2 \pi)^{a-\mu-1}}\left|I_{n, a-1}(a-\mu)\right| \\
= & \frac{n !}{\sqrt{a}(2 \pi)^{(a-1) / 2-\mu} n^{a / 2+1 /(2 a)}} \\
& \quad \times \exp \left(\sum_{m=1}^{a-1} b_{m}(a) \cos \left(\frac{\pi m(a-\mu)}{a}\right) n^{1-m / a}\right)\left(1+O\left(n^{-1 / a}\right)\right),
\end{aligned}
$$

as required.

Similarly, to evaluate the integral $I_{n, \mu}(0)$ we may apply Lemma 4.2 to obtain

$$
I_{n, \mu}(0)=\frac{1}{(2 \pi i)^{a-\mu-1}} \sum_{k=0}^{a-\mu-1}(-1)^{k}\left(\begin{array}{c}
a-\mu-1 \\
k
\end{array}\right) I_{n, a-1}(a-\mu-1-2 k) .
$$


It follows by Lemma 4.3 that if $a-\mu$ is odd, then the quantity $I_{n, \mu}(0)$ represents a linear combination of $I_{n, a-1}(0)$ and sums of the complex conjugates $I_{n, a-1}(u)$ and $\bar{I}_{n, a-1}(u)$ for $u=2,4, \ldots, a-\mu-1$. Similarly, if $a-\mu$ is even, then $I_{n, \mu}(0)$ is equal to a linear combination of differences of the complex conjugates $I_{n, a-1}(u)$ and $\bar{I}_{n, a-1}(u)$ for $u=1,3, \ldots, a-\mu-1$. Now by Lemma 5.3 it is clear that the term with dominant exponent on the right of (6.4) is $\operatorname{Re} I_{n, a-1}(a-\mu-1)$ or $\operatorname{Im} I_{n, a-1}(a-\mu-1)$ depending on whether $a-\mu$ is odd or even.

By Lemma 4.3 we obtain

$$
\begin{aligned}
\operatorname{Re} I_{n, a-1}(a-\mu-1)= & \frac{(2 \pi)^{(a-1) / 2}}{\sqrt{a}} \frac{n !}{n^{a / 2+1 /(2 a)}} \cos (P(n)) \\
& \quad \times \exp \left(\sum_{m=1}^{a} b_{m}(a) \cos \left(\frac{\pi m(a-\mu-1)}{a}\right) n^{1-m / a}\right)\left(1+O\left(n^{-1 / a}\right)\right)
\end{aligned}
$$

and

$$
\begin{aligned}
\operatorname{Im} I_{n, a-1}(a-\mu-1)= & \frac{(-1)^{n}(2 \pi)^{(a-1) / 2}}{\sqrt{a}} \frac{n !}{n^{a / 2+1 /(2 a)}} \sin (P(n)) \\
& \quad \times \exp \left(\sum_{m=1}^{a} b_{m}(a) \cos \left(\frac{\pi m(a-\mu-1)}{a}\right) n^{1-m / a}\right)\left(1+O\left(n^{-1 / a}\right)\right)
\end{aligned}
$$

where

$$
P(n)=\frac{\pi(a-\mu-1)(a-1)}{2 a}-\sum_{m=1}^{a} b_{m}(a) \sin \left(\frac{\pi m(a-\mu-1)}{a}\right) n^{1-m / a} .
$$

Since the sine and cosine functions are bounded, we immediately obtain the required upper bound (6.1). On the other hand, by Weil's theorem, it is possible to show (see Rivoal's argument in the proof of [12, Proposition 13]) that each of the sequences for $\cos (P(n))$ and $\sin (P(n))$ is dense in the interval $[-1,1]$. This implies that there are infinitely many $n$ such that the absolute values of the cosine (sine) are not less than $1 / 2$ and hence the lower bound (6.2) follows for infinitely many $n$.

Lemma 6.2. Let $a \geq 2$ be an integer. Then there exists a positive constant $\lambda=\lambda(a)$ such that for every $\mu=0,1, \ldots, a-1$,

$$
\left|F_{n, \mu}\right| \leq \frac{\lambda n !}{n^{a / 2+1 /(2 a)}} \exp \left(\sum_{m=1}^{a-1}(-1)^{m} b_{m}(a) \cos \left(\frac{2 \pi m}{a}\right) n^{1-m / a}\right) .
$$

Moreover, the asymptotic formula

$$
q_{n}=\frac{n !}{\sqrt{a}(2 \pi)^{(a-1) / 2} n^{a / 2+1 /(2 a)}} \exp \left(\sum_{m=1}^{a}(-1)^{m} b_{m}(a) n^{1-m / a}\right)\left(1+O\left(n^{-1 / a}\right)\right)
$$

holds as $n \rightarrow \infty$. 
Proof. The proof follows easily from Lemmas 4.1, 2.2 and 6.1 and the fact that $q_{n}=F_{n, 0}=I_{n, 0}(1)$.

Our Theorem now follows by Lemmas 2.1 and 6.2.

\section{Acknowledgements}

This work was done during our summer visit in 2010 to the Abdus Salam International Centre for Theoretical Physics (ICTP), Trieste, Italy. The authors wish to thank the staff and, in particular, the Head of the Mathematics Section of the ICTP, Professor Ramadas Ramakrishnan, for their hospitality and the excellent working conditions. The first author is grateful to the Commission on Development and Exchanges of the IMU for travel support.

\section{References}

[1] A. I. Aptekarev (ed.), Rational Approximation of Euler's Constant and Recurrence Relations, Current Problems in Math., 9 (Steklov Math. Inst. RAS, Moscow, 2007).

[2] E. T. Bell, 'Exponential polynomials', Ann. of Math. (2) 35 (1934), 258-277.

[3] L. Comtet, Advanced Combinatorics (D. Reidel, Dordrecht, 1974).

[4] N. G. de Bruijn, Asymptotic Methods in Analysis (Dover, New York, 1981).

[5] M. V. Fedoryuk, Asymptotics: Integrals and Series, Mathematical Reference Library (Nauka, Moscow, 1987).

[6] Kh. Hessami Pilehrood and T. Hessami Pilehrood, 'On a continued fraction expansion for Euler's constant', Preprint, arXiv:1010.1420[math.NT].

[7] Kh. Hessami Pilehrood and T. Hessami Pilehrood, 'Rational approximations for the quotient of Gamma values', Indag. Math. 20(4) (2009), 583-601.

[8] Kh. Hessami Pilehrood and T. Hessami Pilehrood, 'Rational approximations for values of the digamma function and a conjecture on denominators', Mat. Zametki 90(5) (2011), 744-763. English transl.: Math. Notes 90(5) (2011), 730-747.

[9] Y. L. Luke, The Special Functions and Their Approximations, Vol. 1 (Academic Press, New York, 1969).

[10] N. E. Nörlund, "Mémoire sur les polynomes de Bernoulli', Acta Math. 43 (1922), 121-196.

[11] M. Petkovšek, H. S. Wilf and D. Zeilberger, $A=B$ (A. K. Peters, Wellesley, MA, 1997).

[12] T. Rivoal, 'Rational approximations for values of derivatives of the Gamma function', Trans. Amer. Math. Soc. 361 (2009), 6115-6149.

[13] D. N. Tulyakov, 'A system of recurrence relations for rational approximations of the Euler constant', Math. Notes 85(5) (2009), 746-750.

KH. HESSAMI PILEHROOD, Department of Mathematics and Statistics, Dalhousie University, Halifax, Nova Scotia, Canada B3H 3J5

e-mail: hessamik@gmail.com

T. HESSAMI PILEHROOD, Department of Mathematics and Statistics, Dalhousie University, Halifax, Nova Scotia, Canada B3H 3J5

e-mail: hessamit@gmail.com 